\title{
Prevalencia de anemia en adultos mayores no institucionalizados de Lima metropolitana, en relación al nivel socioeconómico
}

\author{
Anemia prevalence in elderly, non-institutionalized adults from \\ metropolitan Lima, according to socioeconomic strata
}

\begin{abstract}
Objective: To determine the prevalence of anemia in elderly adults living in Metropolitan Lima (ML), classified according to socioeconomic status (SES). Methods: We sampled 300 elderly residents in Lima using a household sampling plan with uniform distribution in different SES groups. Hemoglobin $(\mathrm{Hb})$ levels were assessed by photometric analysis of capillary blood samples using the HemoCue ${ }^{\circledR}$ system. Anemia was defined using reference values $\mathrm{Hb}<13 \mathrm{~g} / \mathrm{dL}$ for males and $\mathrm{Hb}<12 \mathrm{~g} / \mathrm{dL}$ for females. Results: The overall $H b$ (mean $\pm S D)$ for men in this elderly population in $M L$ was $13.5 \pm 1.8 \mathrm{~g} / \mathrm{dL}$ compared to $12.7 \pm 1.3 \mathrm{~g} / \mathrm{dL}$ in women. No significant differences in the mean $\mathrm{Hb}$ concentration by sex $(p=0.205)$ were observed. Prevalence of anemia for men and women was $30.5 \%$ and $23.8 \%$. The prevalence of anemia in the low, medium and high SES groups was $26.0 \%$ (17.3-34.7), 29.0\% (20.0-38.0) and 25.0\% (16.4-33.6), respectively, $(p=0.801)$. Conclusions: Anemia prevalence in this elderly metropolitan population was not different among different socioeconomic status groups.

Keywords: Elderly, anemia, hemoglobin, socioeconomic status.
\end{abstract}

\section{INTRODUCCIÓN}

Durante los últimos 50 años, la disminución de la natalidad y la mortalidad en el mundo han conducido, paulatinamente, al envejecimiento de la población. En los países latinoamericanos, entre ellos el Perú, el envejecimiento de la población es una característica demográfica que va adquiriendo relevancia'.

En Perú, en los últimos 35 años, la población de 60 y más años de edad, se ha triplicado en número, al pasar de 798.689 personas $(5,9 \%)$ en 1972 a 2.495 .643 personas $(9,1 \%)$ en el $2007^{2}$. En la actualidad, la población adulta mayor (AM) es de 3 millones 11 mil 50 personas $(9,7 \%)^{3}$. El mayor porcentaje (70\%) de la población AM vive en zona urbana, destacando Lima Metropolitana (LM) con el mayor porcentaje de AM del país $(37 \%)^{2}$.

No obstante, el crecimiento de este grupo poblacional en
Haydee Cárdenas-Quintana ${ }^{1,2}$, Luis Roldan-Arbieto ${ }^{3}$.

1. Departamento de Nutrición, Facultad de Zootecnía, Universidad
Nacional Agraria La Molina, Lima, Perú.
2. Escuela de Postgrado, Universidad Nacional
Federico Villarreal. Lima, Perú.

Dirección para correspondencia: Dra. Haydee Cárdenas-Quintana. Av. La Molina s/n La Molina, Lima, Perú. 00516147800 anexo 485.

E-mail: hcardenasq@lamolina.edu.pe.

Este trabajo fue recibido el 1 de agosto de 2016. Aceptado con modificaciones el 13 de marzo de 2017 y aceptado para ser publicado el 08 de mayo de 2017.

nuestro país, los estudios epidemiológicos y sus determinantes sociales, son aún escasas. Un estudio reciente reportó que los problemas económicos, funcionales y psicosociales influyen negativamente en la salud del AM de $\mathrm{LM}^{4}$.

En el año 2005, se realizó la primera Encuesta Nacional de Indicadores Nutricionales relacionados con enfermedades crónicas de la población adulta peruana ${ }^{5}$. El estudio reportó prevalencia de anemia en el $11,2 \%$ de la población AM, con un promedio de hemoglobina $(\mathrm{Hb})$ de $13,7 \mathrm{~g} / \mathrm{dL}$ (IC 95\% 13,5-13,9).

Tarqui-Mamani y et.al ${ }^{6}$ reportaron que la prevalencia de anemia en el AM del Perú, alcanzó un 23,3\% (Leve $17,1 \%$, moderada $5,7 \%$ y severa $0,5 \%$ ). El mismo estudio indicó que la anemia estuvo asociado a la edad: los AM de 70 a 79 años presentaron un OR 1,5 y los $>80$ años un valor OR 2,1. 
En los últimos años se han documentado estudios epidemiológicos sobre la existencia de anemia en el anciano, los mismos que han demostrado que la anemia es un factor predictivo de mal pronóstico funcional y vital a mediano y largo plazo ${ }^{7-9}$.

La anemia es un problema frecuente en las personas mayores y su prevalencia varia ampliamente, dependiendo del país. Los reportes señalan que el rango de prevalencia se encuentra entre 9 y $11 \%$, valor que se duplica en los mayores de 85 años. Igualmente, los reportes señalan mayor prevalencia de anemia en varones. Los reportes, también, señalan que la anemia se cuadriplica en los ancianos institucionalizados ${ }^{10-13}$.

El proceso de envejecimiento se da en un contexto de pobreza. Estas condiciones influyen, sustancialmente, en el estado nutricional y consumo de alimentos de los $\mathrm{AM}^{12,14}$. La relación entre el nivel socioeconómico (NSE) y el estado nutricional es un tema de discusión y de relevancia en la actualidad. Igualmente, Tarqui-Mamani y et.al ${ }^{6}$ concluyeron que aproximadamente, la cuarta parte de los AM del Perú, presentaron anemia, siendo esta más predominante en los analfabetos, procedentes de áreas rurales y pobres. Sin embargo, el mencionado estudio no contempla la situación de prevalencia de anemia de los AM de LM considerando condiciones socioeconómicas diferenciadas.

El objetivo de este estudio, fue determinar la prevalencia de anemia de adultos mayores de condiciones socioeconómicas diferenciadas en los habitantes de Lima Metropolitana.

\section{SUJETOS Y MÉTODOS}

Población y muestra

Adultos mayores de 60 y más años residentes en viviendas de LM, capaces de responder por sí mismos. Los criterios de exclusión fueron: enfermedad aguda, cambios de peso en los últimos 3 meses, enfermedades graves (cáncer generalizado), enfermedades siquiátricas e intervenciones quirúrgicas recientes (días).

Se seleccionaron $300 \mathrm{AM}$, calculados en base a un muestreo aleatorio simple, con 95\% de confianza, prevalencia de $23,3 \%$ de anemia ${ }^{6}$ y un error de estimación de $5 \%$. Adicionalmente, se consideró una tasa de no respuesta de 10\%. La selección se realizó por nivel socioeconómico (alto, medio y bajo), definidos por IPSOS Apoyo, Opinión y Mercado, que consideran criterios como el presupuesto familiar, características de vivienda y tenencia de bienes y servicios ${ }^{15}$. La distribución de la muestra en cada estrato se hizo mediante la afijación uniforme. En cada estrato se seleccionó a la muestra mediante muestreo por etapas de conglomerados. En la primera etapa se seleccionaron aleatoriamente los distritos; en la segunda etapa, las manzanas (agrupación geográfica de viviendas) de los distritos seleccionados previamente; y en la tercera etapa, las viviendas, donde se seleccionó a un AM por el método de Kish. Con esta selección se logró una mejor cobertura geográfica, así como asegurar la independencia de las observaciones, requisito indispensable para demostrar las hipótesis planteadas en el estudio. A los participantes se les notificaron los objetivos y procedimiento del estudio y se obtuvo su consentimiento informado, el estudio fue aprobado por el Comité de ética de la Universidad Católica de Santa María de Arequipa.

Variables de estudio

Concentración de hemoglobina

Para evaluar el estado de anemia se determinó la concentración de hemoglobina $(\mathrm{Hb} ; \mathrm{g} / \mathrm{dl})$ en una muestra de sangre capilar utilizando un hemoglobinómetro portátil del sistema HemoCue ${ }^{\circledR}$. La prevalencia de anemia se obtuvo usando los puntos de corte a nivel del mar, ya que la ciudad de Lima, se ubica a orillas del mar. Para el caso de varones se utilizó $\mathrm{Hb}:<13 \mathrm{~g} / \mathrm{dl}$ y para las mujeres $\mathrm{Hb}$ : $<12 \mathrm{~g} / \mathrm{dl}$. Se consideró anemia leve $(\mathrm{Hb} \geq 10,0 \mathrm{~g} / \mathrm{dL}$ a $\leq 12,9$ $\mathrm{g} / \mathrm{dL})$, moderada $(\mathrm{Hb} \geq 8,0 \mathrm{~g} / \mathrm{d}$ a $\leq 10,9 \mathrm{~g} / \mathrm{dL}$ ) y severa $(\mathrm{Hb}$ $<8,0 \mathrm{~g} / \mathrm{dL}$ ) en los hombres. También se consideró anemia leve ( $\mathrm{Hb} \geq 11,0 \mathrm{~g} / \mathrm{dL} \mathrm{a} \leq 11,9 \mathrm{~g} / \mathrm{dL}$ ), moderada ( $\mathrm{Hb} \geq 8,0 \mathrm{~g}$ / $\mathrm{dL} \mathrm{a} \leq 10,9 \mathrm{~g} / \mathrm{dL})$ y severa $(\mathrm{Hb}<8,0 \mathrm{~g} / \mathrm{dL})$ en las mujeres ${ }^{16}$.

\section{Análisis estadístico}

Para evaluar la significación de las diferencias en el comportamiento de las variables con el NSE, se utilizó la prueba chi-cuadrado de homogeneidad. Para caracterizar el comportamiento de las variables cuantitativas, se utilizó la media aritmética y la desviación estándar. La significación de las diferencias de su comportamiento entre NSE, se comprobó mediante la prueba de análisis de varianza de una vía. Se utilizó la prueba de Bonferroni para identificar el o los pares de estratos que hacen la diferencia. Para la comparación de variables cuantitativas por sexo se utilizó la prueba t-student de comparación de dos poblaciones. Se consideró una diferencia significativa cuando el valor $\mathrm{p}$ fue menor a 0,05 .

\section{RESULTADOS}

En la tabla 1 se presentan las características de la población en estudio, distribuidos por grupos de edad, género, estado civil y NSE. El 46,7\% (140) de los AM se encontraban entre los 60 a 69 años. El 57,3\% (172) son mujeres.

El valor medio de $\mathrm{Hb}$ en los $\mathrm{AM}$ varones y mujeres fue de $13,5 \pm 1,8 \mathrm{~g} / \mathrm{dL}$ y $12,7 \pm 1,3 \mathrm{~g} / \mathrm{dL}$, respectivamente. El valor medio de $\mathrm{Hb}$ en adultos de 60 a 69 años fue de $13,3 \pm 1,4 \mathrm{~g} /$ dL. Esta cifra es superior a la concentración de $\mathrm{Hb}$ de adultos mayores de 80 años $(12,2 \pm 1,8 \mathrm{~g} / \mathrm{dL})$, valores que presentan diferencias significativas $(p=0,001)$. La concentración promedio de $\mathrm{Hb}$ en función al NSE, no presenta diferencias significativas entre ellas ( $p=0,751$ ) (Tabla 2$)$.

La prevalencia de anemia general fue $26,7 \%$, de los cuales, $18,7 \%$ presentó anemia leve, $7,3 \%$ moderada y $0,7 \%$ severa. La prevalencia de anemia fue de $23,8 \%$ para las mujeres y de $30,5 \%$ para varones, sin diferencia significativa entre sexos $(p=0,205)$. Hay incremento de anemia con el incremento de la edad desde $21,4 \%$ a $41,3 \%$ en los grupos de mayor edad, con diferencias estadísticamente significativas $(p=0,030)$ (Tabla 3$)$. 
TABLA 1

Caracterización de la población adulta mayor de Lima Metropolitana.

\section{Características}

\section{Edad}

$60-69$

$70-79$

80 y más

\section{Sexo}

Mujeres

Varones

NSE

Bajo

Medio

Alto n

140

114

46

172

128

100

100

100
$\%$

46,7

38

15,3

57,3

42,7

33,3

33,3

33,3

Estado civil

Conviviente

$14 \quad 4,7$

Casado

190

63,3

Viudo

67

22,3

Divorciado/separado

18

6

Soltero

11

3,7

Total

300

100

NSE: Nivel socio económico

TABLA 2

Concentración de hemoglobina $(\mathrm{g} / \mathrm{dL})$ en la población adulta mayor de Lima Metropolitana, en función al sexo, edad y estrato socioeconómico

\begin{tabular}{|c|c|c|c|c|c|c|}
\hline & & \multirow[t]{2}{*}{$\mathrm{n}$} & \multirow[t]{2}{*}{$\begin{array}{l}\mathrm{Hb} \\
(\mathrm{g} / \mathrm{dL})\end{array}$} & \multicolumn{2}{|c|}{$\begin{array}{l}\text { Intervalo de } \\
\text { confianza }(95 \%)\end{array}$} & \multirow[t]{2}{*}{$\begin{array}{r}\text { Valor } \\
p^{*}\end{array}$} \\
\hline & & & & Inferior & Superior & \\
\hline Población AM & & 293 & 13,0 & 12,9 & 13,2 & \\
\hline \multirow{3}{*}{ NSE } & Alto & 99 & 13,0 & 12,6 & 13,3 & \multirow[t]{3}{*}{0,751} \\
\hline & Medio & 99 & 13,1 & 12,8 & 13,5 & \\
\hline & Bajo & 95 & 13,0 & 12,7 & 13,3 & \\
\hline \multirow{3}{*}{$\begin{array}{l}\text { Grupo de edades } \\
\text { (años) ** }\end{array}$} & $60-69^{(a)}$ & 140 & 13,3 & 13,1 & 13,6 & \multirow[t]{3}{*}{0,001} \\
\hline & $70-79^{(a)}$ & 109 & 13,1 & 1,7 & 12,7 & \\
\hline & 80 y más ${ }^{(b)}$ & 44 & 12,2 & 11,6 & 12,7 & \\
\hline \multirow[t]{2}{*}{ Sexo } & Mujer & 170 & 12,7 & 12,5 & 12,9 & \multirow[t]{2}{*}{0,000} \\
\hline & Varón & 123 & 13,5 & 13,2 & 13,8 & \\
\hline
\end{tabular}

*Con un nivel de confianza de 95\%. Para las comparaciones por NSE y grupos de edad se usó la prueba de análisis de varianza de una vía (ANOVA) y Bonferroni como prueba post hoc. Para sexo la prueba t-Student.

**La letras representan el resultado de la comparación post hoc (Bonferroni) del ANOVA. La letra diferente representa diferencia significativa respecto a los otros grupos $(p<0.05)$. 
TABLA 3

Prevalencia de anemia (\%) en la población adulta mayor de Lima Metropolitana, en función del sexo, edad y estrato socioeconómico

\begin{tabular}{|c|c|c|c|c|c|c|}
\hline \multirow[b]{3}{*}{ Población AM } & \multirow[b]{4}{*}{ Alto } & \multirow{3}{*}{$\begin{array}{r}n \\
293\end{array}$} & \multirow{3}{*}{$\begin{array}{l}\text { Prevalencia } \\
\text { de anemia } \\
(\%) \\
26,7\end{array}$} & \multicolumn{2}{|c|}{$\begin{array}{l}\text { Intervalo de } \\
\text { confianza (95\%) }\end{array}$} & \multirow[t]{3}{*}{ Valor $\mathrm{p}^{*}$} \\
\hline & & & & Inferior & Superior & \\
\hline & & & & 21,6 & 31,7 & \\
\hline \multirow[t]{3}{*}{ NSE } & & 99 & 25,0 & 16,4 & 33,6 & \multirow[t]{3}{*}{0,801} \\
\hline & Medio & 99 & 29,0 & 20,0 & 38,0 & \\
\hline & Bajo & 95 & 26,0 & 17,3 & 34,7 & \\
\hline \multirow[t]{3}{*}{ Grupo de edades (años) } & $60-69$ & 140 & 21,4 & 14,5 & 28,3 & \multirow[t]{3}{*}{0,030} \\
\hline & $70-79$ & 114 & 27,2 & 18,9 & 35,5 & \\
\hline & 80 y más & 46 & 41,3 & 26,5 & 56,1 & \\
\hline \multirow[t]{2}{*}{ Sexo } & Mujer & 170 & 23,8 & 17,4 & 30,3 & \multirow[t]{2}{*}{0,205} \\
\hline & Varón & 123 & 30,5 & 22,4 & 38,6 & \\
\hline
\end{tabular}

*Con un nivel de confianza de 95\%. Para las comparaciones por NSE y grupos de edad se usó la prueba Chi cuadrado de homogeneidad

y para sexo la prueba t-Student

La prevalencia de anemia es más elevada en los AM del NSE medio de LM (29,0\%), sin embargo, estos valores no fueron diferentes con los AM del NSE bajo y alto $(p=0.801)$.

\section{DISCUSION}

La anemia es un problema frecuente en personas mayores y está relacionada con un aumento de la morbilidad y mortalidad. En nuestro estudio, más de la cuarta parte de los AM habitantes en LM presentaron anemia, cifra que se incrementó con la edad y que afectó mayormente a varones. La prevalencia de anemia severa fue relativamente baja $(0,7 \%)$, sin embargo, es importante señalar que el estudio identificó con mayor riesgo de anemia severa a mujeres $(1,2 \%)$, a mayores de 70 años $(1,8 \%)$ y a los habitantes del NSE bajo $(1,0 \%)$.

La prevalencia de anemia en AM de LM (26,7\%) es bastante similar a valores reportados en poblaciones de AM de Perú $(23,3 \%)^{6}$. Por tanto, los resultados de la presente investigación son consistentes con los reportes de la realidad peruana. Sin embargo, es oportuno resaltar que la prevalencia de anemia del presente estudio, supera ampliamente a los reportes en comunidad anciana de otros países, como es el caso de anemia en Brasil $(7,7 \%)^{17}$, mexicano-americano $(10,4 \%)^{18}$, comunidad de Mashhad, Irán $(12,5 \%)^{19}$ y en una población urbana Coreana $(8,3 \%)^{20}$. Es preciso resaltar que, no obstante la diferencia porcentual de anemia en ancianos de los diferentes países, los reportes del presente estudio identificaron la mayor prevalencia de anemia en varones, situación que fue similar con reportes de Perú ${ }^{6}$ y de otros países ${ }^{18,20,21}$.

La presente investigación proporciona información que la prevalencia de anemia en la población evaluada no encontró una asociación significativa entre pertenecer a un determinado NSE y tener valores diferenciados. La falta de asociación significativa podría indicar que las diferencias de las condiciones socioeconómicas de la población estudiada se homogenizan cuando se trata de habitantes de zona urbana como es el caso de Lima Metropolitana.

La anemia es una condición común y multifactorial entre los adultos mayores, sin embargo, es preocupante la elevada prevalencia de anemia reportada en los ancianos de LM, situación que reflejaría los estilos de vida que adoptaron a lo largo de su existencia. La presente investigación, no tuvo como propósito estudiar la etiología de la anemia, sin embargo, es propicio resaltar que se conoce que la anemia en adultos mayores no está bien caracterizado. Aproximadamente un tercio tiene evidencia de anemia deficitarias o carenciales (hierro, ácido fólico, vitamina B12), otro tercio tiene insuficiencia renal y/o inflamación crónica, y pérdidas gastrointestinales por un proceso inflamatorio o neoplasia en el tracto digestivo y el tercio restante tiene una anemia que es inexplicable ${ }^{13,22}$. En el caso de la población estudiada, un hecho que podría estar contribuyendo a la problemática, sería la anemia de etiología deficitaria, ya que investigaciones recientes en ancianos habitantes de LM, reportaron baja ingesta de hierro en las 3 categorías socioeconómicas $(6,9 \pm 4,5 \mathrm{mg} / \mathrm{d}$ para el NSE bajo; $6,9 \pm 4,4$ $\mathrm{mg} / \mathrm{d}$ para el NSE medio y $7,1 \pm 4,4 \mathrm{mg} / \mathrm{d}$ para el NSE alto) y por tanto las adecuaciones dietarías no cumplieron las recomendaciones oficiales ${ }^{14}$.

Otro hecho, que podría estar contribuyendo a la etiología de anemia, estaría relacionada con la presencia de enfermedades crónicas, al respecto, se ha reportado prevalencia de exceso de peso en el $48 \%$ de la población anciana de LM ( $26 \%$ tenía sobrepeso y $22,3 \%$ eran obesos) sin diferencias significativas entre NSE $(p=0,088)^{14}$. Otro 
estudio poblacional de Perú reportó que un 52\% (IC 95\%: 45,4-58,9) de los AM presentaron síndrome metabólico, lo que indicaría que gran parte de la población en estudio no estaría exenta de estar padeciendo alguna/as enfermedades crónicas (diabetes, hipertensión arterial, enfermedades coronarias, entre otros) ${ }^{23}$ y que podría ser la causa de mayor influencia en la prevalencia de anemia en ancianos como se ha reportado en otros estudios nacionales y de otros países ${ }^{1,11,24-26}$.

Finalmente, los hallazgos de la presente investigación, ponen de manifiesto, por primera vez, que el NSE de los AM no influyó de manera significativa en el estado de anemia de la población, lo que permitiría suponer que la anemia no se debería únicamente al NSE de procedencia, sino también a otros factores determinantes (sociales, físicas y psíquicas) además del método de muestreo y al criterio de clasificación del NSE usado en el presente estudio.

En este contexto, es importante mencionar que Perú vive actualmente un proceso de crecimiento económico sostenido que se reflejaría en poblaciones urbanas que estarían abandonando la pobreza y obteniendo un mayor poder adquisitivo ${ }^{27}$, situación que les permitiría la mayor compra de alimentos y menor actividad física, hecho que podría estar asociada a la elevada prevalencia de exceso de peso de la población estudiada.

Esta situación implica un problema serio de salud pública, ya que la dieta y el estado nutricional tienen gran influencia en la prevención o tratamiento de diversas enfermedades crónicas, cuya incidencia va en aumento en nuestro país. Por tanto, para un análisis global, es necesario explorar otros factores de riesgo distintos a las condiciones socioeconómicas que pudieran influir en el estado de anemia de este grupo vulnerable, a fin de establecer estrategias de prevención y/o focalización de intervenciones adecuadas a la realidad peruana. En conclusión, el estado de anemia de los AM no mostró relación con el NSE.

Agradecimientos: Los autores agradecen a la MSc. Nelly Zavaleta Pimentel, investigadora titular del Instituto de Investigación Nutricional, La Molina, Perú, por la revisión crítica a este artículo. Igualmente, agradecen al MD. Moises Rosas Febres, por los aportes en la traducción del artículo.

\section{RESUMEN}

Objetivo: Determinar la prevalencia de anemia de adultos mayores de condiciones socioeconómicas diferenciadas que habitan en Lima Metropolitana. Metodología: Se seleccionó una muestra aleatoria de 300 adultos mayores (AM) residentes en Lima Metropolitana y de diferentes estratos socioeconómicos (NSE) con afijación uniforme. Para evaluar el estado de anemia se determinó la concentración de hemoglobina $(\mathrm{g} / \mathrm{dl}, \mathrm{Hb})$ en una muestra de sangre capilar utilizando un hemoglobinómetro (HemoCue ${ }^{\circledR}$ ). Para clasificar la anemia se utilizó los siguientes puntos de corte: varones $\mathrm{Hb}<13 \mathrm{~g} / \mathrm{dl}$ y mujeres $\mathrm{Hb}<12 \mathrm{~g} / \mathrm{dl}$. Resultados: El valor medio de $\mathrm{Hb}$ en los $\mathrm{AM}$ varones fue $13,5 \pm 1,8 \mathrm{~g} / \mathrm{dL}$ y en mujeres $12,7 \pm 1,3 \mathrm{~g} / \mathrm{dL}$. No hubo diferencias significativas entre género $(p=0,205)$. La prevalencia de anemia fue del $30,5 \%$ para varones y $23,8 \%$ para mujeres. La prevalencia de anemia en los AM correspondientes al NSE bajo fue de $26,0 \%$ (IC 95\%:17,3-34,7), en el NSE medio $29,0 \%$ (IC 95\%:20,0-38,0), y en el NSE alto 25,0\% (IC 95\%:16,4$33,6)$, diferencias que no fueron significativas $(p=0,801)$. Conclusiones: La prevalencia de anemia de los AM del Lima Metropolitano no mostró una relación con el NSE.

Palabras clave: Adulto mayor, Anemia, Hemoglobina, Nivel socioeconómico.

\section{BIBLIOGRAFIA}

1. Varela L, Chávez H, Herrera A, Méndez F, Gálvez M. Profile of the senior adult of Peru. Developing integrated responses of health care systems for a rapidly aging population INTRA II- PERU. OPS/OMS, 2004.

2. Instituto Nacional de Estadística e Informática. National Census 2007, XI of population and VI of Housing. Lima, Perú. 2008.

3. Instituto Nacional de Estadística e Informática. Situation of the adult population. Lima, Perú. 2015.

4. Cárdenas H. Effect of socioeconomic conditions on nutritional status and its impact on the health of older adults in Metropolitan Lima Metropolitana. Tesis grado de Doctor en Salud Pública. Universidad Nacional Federico Villarreal, Perú, 2007.

5. Ministerio de Salud. Instituto Nacional de Salud. Centro Nacional de Alimentación y Nutrición. National Survey of Nutrition, Biochemical, Socioeconomic and Cultural Indicators related to chronic degenerative diseases. Lima: INS-CENAN; 2006.

6. Tarqui-Mamani C, Sánchez-Abanto J, Álvarez-Dongo D, Espinoza-Oriundo P, Jordán-Lechuga T. Prevalence of anemia and associated factors in elderly peruvians. Rev Perú Med Exp Salud Pública 2015; 32(4): 687-692.

7. Balducci L, Ershler WB, Krantz S. Anemia in the elderly: Clinical findings and impact on health. Critical Rev Oncol Hematol 2006; 58: 156-165.

8. Denny SD, Kuchibhatla MN, Cohen HJ. Impact of anemia on mortality, cognition, and function in community-dwelling elderly. Am J Med 2006; 119: 327-334.

9. Artz AS. Anemia and the frail elderly. Semin Hematol 2008; 45: 261-266.

10. Casals J, Matamoros J. Anemia in the elderly. FMC 2008; 15: 122-131.

11. Kushang P. Epidemiology of anemia in older adults. Seminars in Hematology 2008; 45(4): 210-219.

12. Hulshof K, Brussard JH, Kruizinga AG, Telman J, Lowik MR. Socio-economic status, dietary intake and 10 y trends: the Dutch National Food Consumption Survey. Eur I Clin Nutr 2003; 57(1): 128-137.

13. Urrutia A., Sacanella E.,Mascaro J., Formiga F. Anemia in the elderly. Rev Esp Geriatr Gerontol 2010; 45(5): 291-297.

14. Cárdenas-Quintana H., Roldan L. Relation between nutritional status and socioeconomic level of non-institutionalyzed elderly of Peru. Rev Chil Nutr 2013; 40(4): 343-350.

15. IPSOS Apoyo, Opinión y Mercado. Maps with socio-economic information MAPINSE. Lima Perú, 2005.

16. WHO. Haemoglobin concentrations for the diagnosis of anaemia and assessment of severity. Vitamin and Mineral Nutrition Information System. Geneva, World Health Organization, 2011. 
17. Corona L, Oliveira $Y$, Lebrao M. Prevalence of anemia and associated factors in older adults: evidence from the SABE Study. Rev Saude Publica 2014; 48(5): 723-731.

18. Guralnik J, Eisenstaedt R, Ferrucci L, Klein HG, Woodman $R C$. Prevalence of anemia in persons 65 years and older in the United States: evidence for a high rate of unexplained anemia. Blood 2004; 104: 2263-2268.

19. Eftekharzadeh-Mashhadi I., Hedayati-Moghaddam M., Fathimoghadam F., Bidkhori H., Shamsian S. Anemia as a Public Health Issue in Mashhad, Iran: Evidence from the First Population-Based Study. Acta Medica Iranica 2015; 53(3): 186-190.

20. Bang SM, Lee JO, Kim YJ, Lee KW, Lim S, Kim JH, et al. Anemia and activities of daily living in the Korean urban elderly population: Results from the Korean Longitudinal Study on Health and Aging (KLoSHA). Ann Hematol 2013; 92(1): 59-65.

21. Contreras-Manzano A, De la Cruz V, Villalpando $S$, Rebollar $R$, Shamah-Levy T. Anemia and iron deficiency in Mexican elderly population. Results from the Ensanut 2012. Salud
Publica Mex 2015; 57: 394-402.

22. Guralnik JM, Eisenstaedt RS, Ferrucci L, Klein HG, Woodman RC. Prevalence of anemia in persons 65 years and older in United States: evidence for a high rate of unexplained anemia. Blood 2004; 104: 2263-2268.

23. Cárdenas H, Sánchez J, Roldan L, Mendoza F. Prevalence of Metabolic Syndrome in People 20 Years Old and More. Perú, 2005. Rev Española Salud Pública 2009; 83(2): 257-265.

24. Smith D. Anemia in the elderly. Am Fam Physician 2000; 62(7): 1565-1572.

25. Tettamanti $M$, Lucca U, Gandini F, Recchia A, Mosconi P, Apolone $G$, et al. Prevalence, incidence and types of mild anemia in the elderly: the "Health and Anemia" populationbased study. JHaematologica 2010; 95(11): 1849-1856.

26. Instituto Nacional de Estadística e Informática. Health situation of the elderly adult population. 2012. Lima, Perú. 2013.

27. Vargas E. El impacto del gasto público sobre el proceso de migración interna: Evidencia empírica para el periodo 20022007. Rev Estudios Económicos 2012; 23: 67-81. 